\title{
The capturing of turbulent gas flows using multiple Kinects
}

\author{
K. Berger K. Ruhl M. Albers Y. Schröder \\ Al. Scholz J. Kokemüller S. Guthe M. Magnor \\ Computer Graphics Lab \\ TU Braunschweig \\ \{berger, ruhl, albers, schroeder, scholz, kokemueller, magnor, guthe\}@cg.cs.tu-bs.de
}

\begin{abstract}
We introduce the Kinect as a tool for capturing gas flows around occluders using objects of different aerodynamic properties. Previous approaches have been invasive or require elaborate setups including large printed sheets of complex noise patterns and neat lighting. Our method is easier to set up while still producing good results. We show that three Kinects are sufficient to qualitatively reconstruct nonstationary time varying gas flows in the presence of occluders.
\end{abstract}

\section{Introduction}

In a typical gas flow or turbulance flow visualization setup, the object under investigation is immersed into gas flows which are enhanced with small seeding particles. These particles provide contrast to the background and when moved inside the flow they act as streamlines that characterize the flow field at discrete positions. However, the immersion of these particles always causes small disturbances in the gas flow to be captured. Besides, for each two adjacent frames showing the projection of the particles, the correspondence estimation between the projected particles has to be solved. This tedious task can be alleviated by introducing particles of different color [5] or by allowing longer exposure times [17].

A different non-invasive approach relies on the light path deviations introduced on illuminated background patterns (BOS, or background oriented Schlieren technique). In this approach, a complex pattern has to be printed on a large sheet of paper and has to be placed behind the scene. The pattern is captured once without the gas flow and then for each frame with a gas flow present in the scene [3]. Using optical flow or cross-correlation the projected deviations are then used to characterise the captured flow. While this approach provides the most authentic measurement of the gas stream, it still suffers from the fact that a lot of effort has to be put into designing the background pattern [1] and con- sidering the distance between camera and the background pattern. Furthermore, the amount of printed paper sheets for realising the background pattern technique is not negligible.

With the advent of the Kinect [14] and its combination of a passive and active-light sensor, several opportunities arise to overcome the current problems. First, the Kinect accurately provides a distance predication for walls or projections planes to which they are opposed to. Second, the Kinect uses an elaborate robust pattern that can be projected up to 7 meters without a loss in quality.

In this paper, we propose to use the Kinect as a new sensor to non-invasively capture the gas flows around objects that are introduced into the flow. We provide qualitative images of the gas flow alterations on the projected IR light patterns and 4D reconstructions of the gas streams around objects.

The remainder of the paper is organised as follows: We start with a short overview of the state of the art in Sec. 2. Then we introduce the capturing setup consisting of multiple Kinects and a versatile method to calibrate them to a common world space in Sec. 3. Next, we provide the theoretical background necessary to understand our reconstruction method in Sect. 4 and we perform the deviation computation and reconstruction in Sec. 5. Finally, we present the reconstructed results, in Sec. 6, before we conclude in Sec. 7.

\section{Related Work}

Capturing turbulent flows or flows in general is a longstanding research area. Kasai et al. [13] imaged the blood flow in organs by employing cross correlation techniques on laser doppler echoes at different time instants. Another approach by Prasad and Sreenivasan [16] produces scalar fields of turbulent flows exiting a jet nozzle by employing an energy minimization on the pixel intensity of captured flourescent fluid in water using block matching. The flourescence is induced by a laser. A rather new approach on the basis of Magnetic Resonance Imaging (MRI) 
concerned with the capturing of wakes and turbulances was introduced by Newling et al. [15]. However, all methods can be either categorized as invasive, using seeding particles, or non-invasive, relying on laser-doppler-effects or elaborated pattern analysis.

\section{Invasive techniques}

A prominent invasive aproach is called the particle imaging velocimetry (PIV). It relies on seeding particles into the flow under examination. The extension to tracing flowing particles in consecutive images is called particle tracing velocimetry (PTV). A method to apply PTV of a turbulent flow in 3D has been presented by Doh et al. [8]. Still, given only the images of two adjacent time frames, estimating the correlation of a multitude of particle points is an ill-posed problem. Bordas et al. [5] proposed to use differently coloured particles to ease the matching.

\section{Non-invasive techniques}

Voigt et al. [18] present a technique to use the laser-doppler effect for imaging the velocity components of a flow on the basis of 2D images. Another approach is based on the flow-based light path alteration of elaborated background patterns that result in different projection images. Atcheson et al. [2] proposed a method to capture the time-resolved motion of gas flows exploiting the BOS technique. A further enhancement to also capture flows around occluding objects was proposed by Berger et al. [3]. However, they had to carefully engineer a robust background pattern [1] and used a widely-spaced setup where the capturing quality depended on the amount of indirect illumination.

Our method using a Kinect in a simple setup is a noninvasive technique that does not depend on elaborated background-patterns and indirect illumination.

However as we employ a setup consisting of multiple Kinects, i.e. multiple interfering active light sensors, we have to take interference and calibration to a common world space into account.

\section{Multiple Kinect Setups}

As the Kinect is a combined passive RGB and an active structured light sensor, simultaneous calibration has the same challenges as previous fusion approaches, as e.g. by Gudmundsson et al.[11] or Huhle et al. [12]. However, both used a fixed rig while we employ a static but otherwise freely configurable setup. On the internet, several interesting approaches have been proposed to put the Kinect into a multi-camera setup, ranging from finding texture differences in the IR sensor with an occluded emitter [9] to treating the checkerboard printout as a planar surface [7]. Another approach uses 3D-printouts of cuboids to provide distinguishable values in the depth image [10]. However these solutions remain ad-hoc and have not been
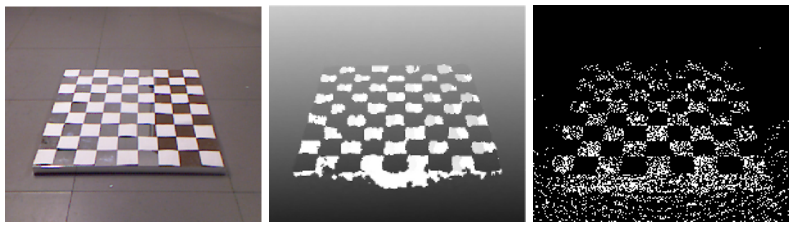

Figure 1. We use the approach by Berger et al. [4], that solves the problem that RGB and depth sensors cannot be calibrated simultaneously. Thus, we use binary surface patterns, e.g. a checkerboard consisting of white diffuse and mirroring patches (left). In the depth (middle) and IR image (right, thresholded for better visibility) the pattern becomes clearly distinguishable.

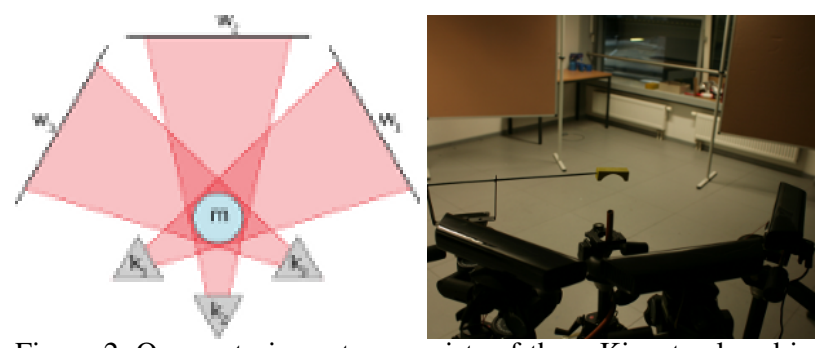

Figure 2. Our capturing setup consists of three Kinects placed in a small half circle with an angular spacing of $45^{\circ}$ between each other (left diagram). The Kinects $k_{i}$ capture opposite planes $w_{i}$ using both the passive rgb sensor and the active IR emitter. The refractive medium $m$, e.g. the propane gas plume of refractive index 1.3407 distorts the emitted patterns providing cues in the depth images. Right: a setup with three Kinects and projection walls, the gas nozzle an an occluder. Note, that the only constraint imposed on the planes is that they need to be flat and diffuse. No binary pattern printout is needed.

proven in setups with multiple Kinects. We found that the simultaneous calibration of a passive and an active sensor can be solved well by employing materials with different BRDFs. To this end, we used a mirror-diffuse checkerboard pattern, shown in Fig. 1 and further discussed in Sec. 3.2

\section{Setup and Calibration}

Our setup involves three Kinects, each facing a wall perpendicular to its viewing axis. The three Kinect sensors involved are calibrated into a common world space with the help of a mirror-diffuse checkerboard.

\subsection{Setup}

We conduct our turbulence flow studies in a lab room environment measuring $5 m \times 5 m \times 2.5 m$, Fig. 2 . The source of the gas flow, a high pressure propane gas nozzle (pressure $\approx 400 \mathrm{kPa}$ ), is placed in the center of the room together with three Kinects spanning a small quarter-arc around it. Commodity propane has the refractive index of 1.3407 , the fourth-greatest index for gases, thus it provides the necessary light path deviation needed to distinguish the gas plume from the surrounding air as the refractive index is roughly 
a)

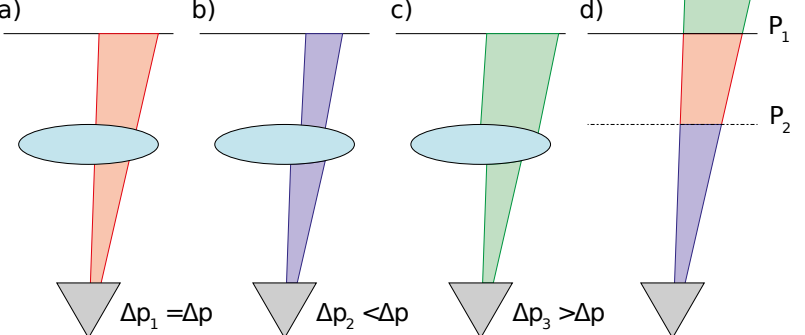

Figure 3. Structured light ray path deviations induced by a gas with significantly different refractive index $(a, b, c)$ equal perceived virtual planes at varying depth in the depth sensor (d).

1.0002 at $15^{\circ} \mathrm{C}$ room temperature in the depth images. For each Kinect, we provide a projection plane placed at a defined distance of $3.04 \mathrm{~m}$. The plane's rotation is optimized so that the mean pixel value difference for pixels backprojected on that plane is minimized. Initially we also provided diffuse indirect lighting from above the room, but it proved to be unnecessary, further reducing setup efforts.

\subsection{Checkerboard-Calibration}

In order to simultaneously align the three Kinects, i.e. three RGB sensors and three IR sensors, we use the new calibration pattern as proposed by Berger et al. [4]. Our goal is to provide a clear and distinguishable pattern in both the RGB sensors and three IR sensors.

We use a checkerboard pattern. A normal checkerboard pattern, printed out on paper, however, will uniformly reflect the IR pattern back to the Kinects and thus the captured image of the checkerboard only consists of indistinguishable depth values, at most a gradient. Berger et al. [4] found that mirroring materials, placed at the right angle (i.e. every angle except nearly orthogonal to camera's viewing axis), will project the patterns to infinity. Therefore, we used a checkerboard pattern that is white and diffuse on the one hand and mirroring on the other hand, Fig. 1 (left), thereby getting a projected checkerboard image in both the RGB and IR sensor, Fig. 1 (right), which can be used for robust alignment. The resulting images are processed with the Matlab calibration toolbox [6].

\section{Mathematical Background}

As shown by Atcheson et al. [2], a gas with a significantly different refractive index than the surrounding air introduces small deviations on the light path $\vec{r}$ of a ray traveling through it. That method has been exploited in Schlieren imaging and Schlieren tomography. There, the ray has formed at the background pattern which is diffusely illuminated. In our setup we actively emit an IR pattern that travels through the refractive gas. The effects on a captured depth image can be reasoned as follows:

Consider two pixels of the projected IR pattern, whose projected pixel difference in the IR sensor would be $\Delta_{P}$ when reflected from a plane $P$ at distance $d$, parallel to the image plane of the depth sensor. Now these two pixels correspond to two rays $\overrightarrow{r_{1}}, \overrightarrow{r_{2}}$, that travel through a gas with a significantly different refractive index than the surrounding air. three cases can occur:

1. The ray paths are not altered (Fig. 3 a). This is true for a negligible index gradient between the gas and air or for rays that travel orthogonally through the boundary layer of the gas.

2. The ray paths are bent towards each other (Fig. 3 b).

3. The ray paths are bent away from each other (Fig. 3 c).

The cases two and three depend on the incident angle of the light rays at the boundary layer between the gas and the air. In case 1 , the projected pixel difference in the IR sensor would be $\Delta_{P_{1}}$ and would equal $\Delta_{P}$ (Fig. $3 \mathrm{~d}$, red plane). In case 2 , the projected pixel difference in the IR sensor would be $\Delta_{P_{2}}$ and would be smaller than $\Delta_{P}$. The disparity would correspond to a parallel plane $P_{2}$ at distance $d_{2}<d$ in the depth image (Fig. 3 d, blue plane). In case 3 , the projected pixel difference in the IR sensor would be $\Delta_{P_{3}}$ and would be greater than $\Delta_{P}$. The disparity would correspond to a parallel plane $P_{3}$ at distance $d_{3}>d$ in the depth image, (Fig. 3 d, green plane).

We want to exploit this fact for our qualitative gas flow capturings, by searching for exactly the depth image deviations $\Delta_{P_{2}}$ and $\Delta_{P_{3}}$ in a depth image captured from a still scene with the gas flow being the only dynamic scene element. We do in fact not discriminate between $\Delta_{P_{2}}$ and $\Delta_{P_{3}}$, since both cases indicate the presence of a light path alteration at the corresponding pixel.

Note, that we only search for these deviations above the projected gas nozzle, not at its casted shadow, which is also present in the depth image.

\section{Qualitative Capturing of turbulent gas flows around objects}

We capture objects with varying drag coeeficients placed in the propane gas stream. The projection planes are calibrated to maximize measurements of the resulting deviations. Finally, the deviations in the depth images $D_{1,2,3}$ are used to generate masks for reconstructing the visual hull of the gas.

\subsection{Captured Objects}

We captured the gas flow around the following objects: A bridge $\left(c_{d}>1.0\right)$, a cylinder $\left(c_{d}=0.82\right)$, a box $\left(c_{d}=\right.$ 


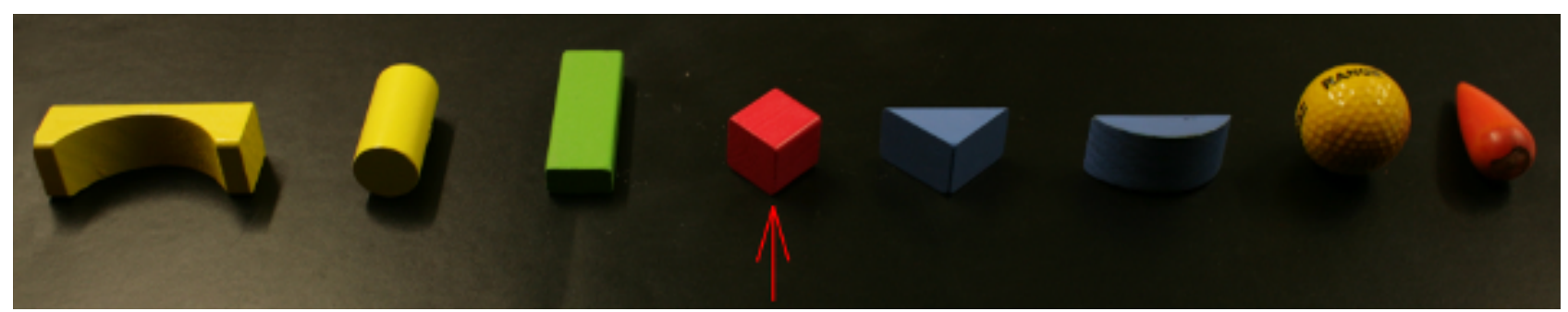

Figure 4. We examined several geometric objects with different aerodynamic properties: bridge $\left(c_{d}>1.0\right)$, cylinder $\left(c_{d}=0.82\right)$, box $\left(c_{d}=0.82\right)$, cube $\left(c_{d}=0.80\right)$, prism $\left(c_{d}=0.50\right)$, half sphere $\left(c_{d}=0.42\right)$, gulf ball $\left(c_{d}=0.3\right)$ and droplet $\left(c_{d}=0.04\right)$. The objects are shown in the same way as they placed into the gas flow from above (indicated by red arrow).

$0.82)$, a cube $\left(c_{d}=0.80\right)$, a prism $\left(c_{d}=0.50\right)$, a half sphere $\left(c_{d}=0.42\right)$, a golf ball $\left(c_{d}=0.3\right)$ and a droplet $\left(c_{d}=\right.$ 0.04). They are placed in the gas stream as shown in Fig. 4.

\subsection{Projection Plane Alignment}

For the capturing of small deviations in the light path induced by gas flows, it is crucial to assume a perfectly calibrated projection plane against which we compare the deviations in the light path. In previous work [2], the cameras have been arranged in an half-arc around the gas flow, while their projection planes have been arranged in the opposite half-arc. This means cameras and projection planes have been aligned roughly to each other. In our setup we want to optimize the alignment of the projection plane to the cameras' viewing axes. To this end, we sample the Kinect's depth image at five positions, the center positions and the four corner positions. We then compute the largest absolute difference $\delta_{c c}$ between the corner values and the center value. Each Kinect $k_{i}$ and its opposite projection plane $w_{i}$ are then fine-rotated to minimize on $\delta_{c c}$. Furthermore the distance between each Kinect $k_{i}$ and its projection plane $w_{i}$ is adjusted to a defined value, in our case $3.04 \mathrm{~m}$. With this, we provide a very accurately calibrated setup for the capturing of deviations.

\subsection{Image Space Preprocessing}

For each Kinect $k_{i}$ we obtain qualitative flow images by performing a background substraction in the depth image, i.e. we capture the scene with no present gas flow to an average image $D_{i}(0)$, and then substract it for each frame $t$ from a captured image $D_{i}(t)$ to get the signed difference image $D_{\text {diff,i }}(t)$, which we may threshold to eliminate sensor noise.

$$
D_{\text {diff,i }}(t)=D_{i}(t)-D_{i}(0), i \in\{1,2,3\}
$$

We then create conservative masks for the projected areas that are comprised by the gas flow by searching for the large connected components $D_{\text {diff, }}(t)$. The projected area comprised by the occluding object is set to be outside the mask. We find the object by searching for pixels very small or invalid depth values in $D(t)$ and mark them in $D_{\text {diff,i }}(t)$ accordingly.

\subsection{D Reconstruction}

We perform the hull reconstruction of the gas flow for frame $t$ similar to Berger et al. [3]. We first reconstruct the visual hull $V H_{\text {gas+object }}$ of the voxels, that include both the object and the gas followed by the visual hull of only the object $V H_{\text {object }}$. With constructive solid geometry, we substract $V H_{\text {object }}$ from $V H_{\text {gas+object }}$ to arrive at $V H_{\text {gas }}$. This way, we ensure that the reconstructed gas hull fits tightly around the object.

\section{Results}

We found that the projection planes could be calibrated in distance up to the exact depth value. We used movable walls and fixed them at a depth value of 999 , which is $3.04 \mathrm{~m}$. The projection planes could be optimized in rotation, so that the maximal difference $\delta_{c c}$ was \pm 1 depth units for a projected area of $200 \times 250$ pixel. In the following, we show the results for different aerodynamic objects. We placed the gas nozzle $\approx 2 \mathrm{~cm}$ below the occluding object and let it vent at $400 k P a$. Tab. 1 lists the difference images for three adjacent time steps and the corresponding visual hull reconstructions for the same time instants and objects. Notice, that the droplet (bottom row) introduces the least turbulences to the gas flow. A consistent flow is noticeable for varying height. Other objects like the cylinder (second row) or the box (third row) seem to block most parts of the gas stream, while objects like the cube (fourth row) or the half sphere (sixth row) force the stream to flow around at one of two possible sides. Also notice, how the bridge blocks the gas and forces it to flow around.

\section{Conclusion}

We introduced the Kinect as a versatile tool to capture and visualize gas flows around objects with varying aerodynamic properties. Light path deviations on the projected pattern introduced by the gas flow can be exploited to determine the projected areas in the images that are covered 


\begin{tabular}{|c|c|c|c|c|c|c|c|}
\hline Object & Drag coefficient & $D_{\text {diff }, 2}(t)$ & $V H_{\text {gas }}(t)$ & $D_{\text {diff, } 2}(t+1)$ & $V H_{\text {gas }}(t+1)$ & $D_{\mathrm{diff}, 2}(t+2)$ & $V H_{\text {gas }}(t+2)$ \\
\hline Bridge & $>1.0$ & $\ldots$ & & $\cdots$ & & $\cdots$ & \\
\hline Cylinder & 0.82 & $\cdots$ & & & & & \\
\hline Box & 0.82 & & & & & & \\
\hline Cube & 0.80 & & & $\cdots$ & & & \\
\hline Prism & 0.50 & $\ldots$ & & $\ldots$ & & & \\
\hline Half sphere & 0.42 & & & & & & \\
\hline Golf ball & 0.3 & & & & & & \\
\hline Droplet & 0.04 & 1 & & 1 & & -- & \\
\hline
\end{tabular}

Table 1. The qualitative results in the difference depth images $D_{\text {diff,2 }}$ and the corresponding visual hull reconstructions of the gases $V H_{\text {gas }}$, captured with 3 Kinects spaced in a quarter-arc around the occluder. The colors indicate positive or negative deviation in the depth images, occluders are marked in maroon color. The volumetric extend of the gas is visualized in grey and rendered from a novel viewpoint. Note the turbulence introduced by objects with a high drag coefficients compared to the streamlined flow around the droplet. 
by the gas. We then generated the visual hull to visualize a time-dependend flow and the turbulences introduced by the objects in three dimensions. However, the results of the introduced flow turbulences remain qualitatively. In the future, we want to investigate quantitatively the light path deviations with tomographic reconstruction methods based on the sparse set of input images.

\section{Acknowledgements}

Funding by the European Research Council ERC under contract No. 256941 "Reality CG" and by the German Science Foundation DFG MA 2555/1-3 is gratefully acknowledged.

\section{References}

[1] B. Atcheson, W. Heidrich, and I. Ihrke. An evaluation of optical flow algorithms for background oriented schlieren imaging. Experiments in fluids, 46(3):467-476, 2009. 1, 2

[2] B. Atcheson, I. Ihrke, W. Heidrich, A. Tevs, D. Bradley, M. Magnor, and H.-P. Seidel. Time-resolved 3d capture of non-stationary gas flows. ACM Transactions on Graphics (Proc. SIGGRAPH Asia), 27(5):132, 2008. 2, 3, 4

[3] K. Berger, I. Ihrke, B. Atcheson, W. Heidrich, M. Magnor, et al. Tomographic $4 \mathrm{~d}$ reconstruction of gas flows in the presence of occluders. In Vision, Modeling, and Visualization Workshop (VMV), Braunschweig, Germany, pages 1-8. Citeseer, 2009. 1, 2, 4

[4] K. Berger, K. Ruhl, Y. Schroeder, C. Brümmer, A. Scholz, and M. Magnor. Markerless motion capture using multiple color-depth sensors. In Proc. Vision, Modeling and Visualization (VMV) 2011, 2011. 3

[5] R. Bordás, C. Bendicks, R. Kuhn, B. Wunderlich, D. Thévenin, and B. Michaelis. Coloured tracer particles employed for 3-d ptv in gas flows. In ISFV13-13th International Symposium on Flow Visualization, and FLUVISU1212th French Congress on Visualization in Fluid Mechanics, Paper, volume 93, pages 1-4. 1, 2

[6] J. Bouguet. Camera calibration toolbox. See http://www. vision. caltech. edu/bouguetj/calib_doc, 2010. 3

[7] N. Burrus. http://nicolas.burrus.name/ index.php/Research/KinectCalibration, November 2010. 2

[8] D. Doh, T. Hwang, and T. Saga. 3d-ptv measurements of the wake of a sphere. Measurement Science and Technology, 15:1059, 2004. 2

[9] N. Engelhard. http://www.informatik. uni-freiburg.de/ engelhar/calibration. html, mai 2011. 2

[10] M. Gaffney. http://www.thingiverse.com/ thing:7793, 2011.2

[11] S. Gudmundsson, R. Larsen, H. Aanaes, M. Pardas, and J. Casas. Tof imaging in smart room environments towards improved people tracking. In Computer Vision and Pattern Recognition Workshops, 2008. CVPRW'08. IEEE Computer Society Conference on, pages 1-6. IEEE, 2008. 2
[12] B. Huhle, P. Jenke, and W. Straßer. On-the-fly scene acquisition with a handy multi-sensor system. International Journal of Intelligent Systems Technologies and Applications, 5(3):255-263, 2008. 2

[13] C. Kasai, K. Namekawa, A. Koyano, and R. Omoto. Realtime two-dimensional blood flow imaging using an autocorrelation technique. IEEE Trans. Sonics Ultrason, 32(3):458464, 1985.1

[14] Microsoft Corporation. Kinect for xbox 360, November 2010. Redmond WA. 1

[15] B. Newling, C. Poirier, Y. Zhi, J. Rioux, A. Coristine, D. Roach, and B. Balcom. Velocity imaging of highly turbulent gas flow. Physical review letters, 93(15):154503, 2004. 2

[16] R. Prasad and K. Sreenivasan. Quantitative threedimensional imaging and the structure of passive scalar fields in fully turbulent flows. Journal of Fluid Mechanics, 216(1):1-34, 1990. 1

[17] A. Sellent, M. Eisemann, and M. Magnor. Motion field and occlusion time estimation via alternate exposure flow. In Computational Photography (ICCP), 2009 IEEE International Conference on, pages 1-8. IEEE. 1

[18] A. Voigt, C. Skupsch, J. Koenig, K. Shirai, L. Buettner, and J. Czarske. Laser doppler field sensor for two dimensional flow measurements in three velocity components. Imaging Measurement Methods for Flow Analysis, pages 21-30, 2009. 2 